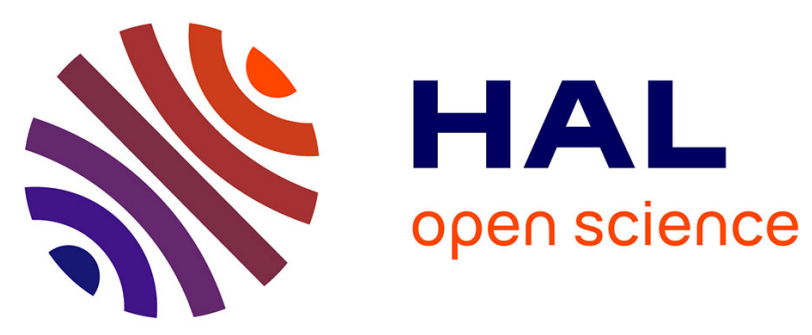

\title{
A Macroscopic Model for Freeway Weaving Sections
}

Florian Marczak, Ludovic Leclercq, Christine Buisson

\section{To cite this version:}

Florian Marczak, Ludovic Leclercq, Christine Buisson. A Macroscopic Model for Freeway Weaving Sections. Computer-Aided Civil and Infrastructure Engineering, 2015, Advanced Computational Methods for Traffic Modeling, Data Processing and ITS, 30 (6), pp.464-477. 10.1111/mice.12119 . hal-01215745v2

\section{HAL Id: hal-01215745 \\ https://hal.science/hal-01215745v2}

Submitted on 21 Apr 2017

HAL is a multi-disciplinary open access archive for the deposit and dissemination of scientific research documents, whether they are published or not. The documents may come from teaching and research institutions in France or abroad, or from public or private research centers.
L'archive ouverte pluridisciplinaire HAL, est destinée au dépôt et à la diffusion de documents scientifiques de niveau recherche, publiés ou non, émanant des établissements d'enseignement et de recherche français ou étrangers, des laboratoires publics ou privés. 


\title{
A macroscopic traffic model for freeway weaving sections
}

\author{
Florian Marczak, Ludovic Leclercq, Christine Buisson
}

Université de Lyon, IFSTTAR/ENTPE

Laboratoire d'Ingénierie Circulation Transports, LICIT

Rue Maurice Audin, 69518 Vaulx en Velin, France

\begin{abstract}
The objective of this paper is to propose an analytical traffic model adapted to freeway weaving sections. To this aim, a theoretical weaving section is considered at the macroscopic level as the superposition of two merges and two diverges. The model endogenously incorporates the capacity drop related to the weaving lane changes. It only depends on six parameters: three parameters of the fundamental diagram (the free-flow speed, the jam density and the wave speed in congestion), the vehicles' acceleration, the relaxation factor and the length of the anticipation zone. A sensitivity analysis proves that the acceleration rate and the relaxation parameter most influence the capacity drop. The analytical model agrees well with microsimulation results and empirical data collected on a weaving section in France.
\end{abstract}

\section{INTRODUCTION}

Weaving areas are the crossing of two or more traffic streams traveling in the same direction. An intense lane changing activity occurs therefore at weaving sections. The lane changes and the complex interactions between the weaving and non-weaving vehicles affect the operation and reduce the capacity of weaving sections in relation to their equivalent basic freeway segments.

Traffic in weaving sections has been examined through data analysis. Using oblique cumulative vehicle counts from loop detectors at two different weaving sections in the USA, Lee and Cassidy (2009) and Skabardonis and Kim (2010) show that the bottleneck activation may be triggered by disruptive lane changes from the ramp to the freeway. The effects of the weaving lane changes are further studied in (Rudjanakanoknad and Akaravorakulchai, 2011) with video data collected on a weaving section in Bangkok. The authors prove that the capacity of the studied weaving section fluctuates over time depending on the number of lane changes and

\footnotetext{
*To whom correspondence should be addressed. E-mail: ludovic.leclercq@entpe.fr.
} 
their destination. They observed that an increase of the on-ramp demand induces lane changes from slow to fast lanes and raises consequently the total capacity. They pinpointed also that an augmentation of the off-ramp demand induces lane changes from fast to slow lanes and reduces the capacity of the studied weaving section. Such interactions between weaving vehicles are investigated at a microscopic level in (Sarvi et al., 2011). The authors exhibit that the acceleration-deceleration behavior and the decision to change lane of the weaving vehicles are influenced by the surrounding freeway vehicles. More recently, Marczak et al. (2014) analyzed detailed microscopic trajectory data collected on a weaving section in Grenoble (France). The authors prove that the lane changing behavior depends strongly on the prevailing traffic conditions. When the traffic is saturated the lane changes occur at the beginning of the weaving section independently of their direction. When the traffic conditions are more fluid, the lane changing positions are more distributed along the weaving section. But interestingly, the lane changes still occur at the same location independently of their direction.

The performance of weaving areas has been also estimated through analytical procedures. The model described in (Lertworawanich and Elefteriadou, 2003) expresses the capacity of a weaving section as a function of the proportions of origin-destination demands and the speeds of the weaving and non-weaving vehicles. The current HCM 2010 methodology (TRB, 2010) to design weaving sections is an update of the HCM 2000 methodology (TRB, 2000) incorporating the improvements developed as part of the NCHRP 3-75 project (Roess and Ulerio, 2009a,b). The capacity of weaving sections is directly linked to the number of lane changes expressed as the percentage of the vehicles that desire to make a weaving movement and the length of the weaving section. However, significant differences may exist between empirical observations of weaving sections capacity and estimates from analytical procedures (Cassidy et al., 1989; Rakha and Zhang, 2006).

Some authors consider microsimulation to be more suitable and reliable to estimate the capacity and predict the operation of various weaving sections. Skabardonis (2002) calibrates CORSIM with field data measured on eight weaving sections with different configurations. The calibrated CORSIM predicts accurately the operation of the different weaving sections for all configurations and demand patterns. Calvert and Minderhoud (2012) generalize the approach initiated originally in (Dijker and Schuurman, 2003). The authors test different weaving configurations with SIMONE, a microscopic traffic model, and derive, from the microsimulation results, the corresponding expression of the capacity as a function of the weaving flow rate.

Some previous works deal specifically with the development of models to reproduce the interactions between the weaving vehicles. Those interactions are modeled using intelligent agent concepts (Hidas, 2005), generic continuous gas-kinetic traffic flows model (Ngoduy, 2006) or gap-acceptance theory (Bahm, 2011). 


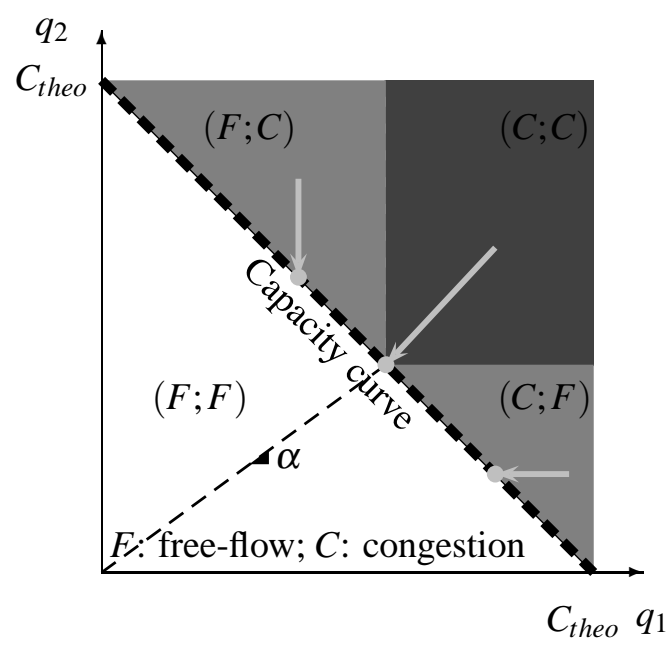

Figure 1: Global allocation scheme for the Newell-Daganzo's model

None of the papers mentioned above explicitly integrates the microscopic behaviors of the drivers to derive an accurate estimation of a weaving section effective capacity. To address this issue, the objective of the present research is to propose a macroscopic model for a freeway weaving section with an allocation scheme similar to Newell-Daganzo model for merges (Daganzo, 1995). Figure 1 is a particular representation of the Daganzo model where the capacity of both incoming links is equal and equal to the downstream capacity of the merge (denoted $C_{t h e o}$ ). The x-axis and the y-axis give the effective flows on the main road and the on-ramp, respectively. In the $(F ; F)$-zone, the merge is free flowing because the demands are lower than the capacity. In the $(C ; F)$-zone all the demand from the on-ramp succeeds in merging while the main road is congested. In the $(F ; C)$-zone the on-ramp is congested while the main road is free flowing. Finally, in the $(C ; C)$-zone the on-ramp and the main road are congested and the effective flows share the downstream available capacity according to a fixed merge ratio $\alpha$.

The proposed model should also incorporate an endogenous expression of the capacity drop. It is postulated that either the slowdowns or the accelerations of the weaving vehicles create voids in the traffic stream that reduce the capacity of the weaving section. It is assumed moreover that there are no spillbacks from bottlenecks downstream of the weaving section. At the macroscopic level, we consider the weaving section as the superposition of two merges and two diverges. We extend the analytical model proposed in (Leclercq et al., 2011) for the merges by explicitly incorporating (i) the effects of the relaxation at a macroscopic level and (ii) the lane changers' behavior in relation to the prevailing traffic conditions on the target lane. We also adapt the model initially proposed in (Laval, 2009) to derive analytically the effective capacity of the diverges.

In (Sarvi and Kuwahara, 2007), it is reported that none of the most frequently used commercial tools can correctly reproduce the traffic behavior near discontinuities of the highway network, especially in congested situations. Previous analytical models mostly fit the outcome of extensive microsimulations. Their results are therefore questionable. Our model is built on a rigorous empirical analysis of the lane changing behavior. The 
previsions of the model are therefore more accurate because they integrate explicitly the real traffic behavior. Unlike the microsimulation models, the proposed analytical model will require few parameters. Moreover, those parameters will have a physical interpretation, and hence can be easily calibrated with field data. The proposed model will provide a direct estimate for the effective capacity of a weaving section without requiring any complex simulation runs or elaborated calibration procedure (Ngoduy, 2011). It also has operational applications. It could be the basis of a tool for traffic road managers to forecast in real time the operation of a weaving section or evaluate dynamic traffic management strategies such as ramp metering (Zhang and Wang, 2013). The estimated capacities could be also incorporated in a dynamic route choice model $(\mathrm{Ng}$ and Waller, 2012) to guarantee better previsions.

The remainder of the paper is structured as follows. A description of the assumptions and the modelling approach are given in Section 2. To quantify the influence of the model parameters, a sensitivity analysis is performed in Section 3. Section 4 finally presents a comparison between the model estimations, empirical observations and microsimulation results.

\section{MACROSCOPIC MODELING OF A WEAVING SECTION}

\subsection{Preliminary assumptions and notations and modeling approach}

We consider a one-sided weaving section with one lane on the main road and one auxiliary lane. Lane 1 is assumed to be the main road while lane 2 is assumed to be the auxiliary lane. Each lane obeys a triangular fundamental diagram with a free-flow speed $u$, a jam density $\kappa$ and a wave speed in congestion $w$. The theoretical capacity of each lane is noted $C_{\text {theo }}$. $C_{\text {theo }}$ is measured in vehicles/time and equals:

$$
C_{\text {theo }}=\frac{u w \kappa}{u+w}
$$

The demand upstream of the main road (resp. the auxiliary lane) equals $\lambda_{1}$ (resp. $\lambda_{2}$ ). $q_{1}$ (resp. $q_{2}$ ) is the effective flow coming from the main road (resp. the auxiliary lane) and crossing the weaving section. $\beta_{1}$ and $\beta_{2}$ are respectively the percentages of weaving vehicles driving from and toward the main road. $\lambda_{i}$ and $\beta_{i}$ $(i \in \llbracket 1,2 \rrbracket)$ are the inputs of the model while $q_{i}(i \in \llbracket 1,2 \rrbracket)$ are the outputs of the model, see Figure 2.

We also introduce a constant acceleration rate $a$. The headways are the time intervals between two successive vehicles. They are assumed to follow a shifted exponential distribution (Gattuso et al., 2005; Chevallier and Leclercq, 2007) upstream of the weaving section. The headways density function on lane $i$ is thus given 
by:

$$
f_{H}^{i}(h)= \begin{cases}\delta_{i} e^{-\delta_{i}\left(h-h_{x}\right)} & \text { if } h_{x} \leq h \\ 0 & \text { otherwise }\end{cases}
$$

Where $h_{x}$ is the minimum safety time headway:

$$
h_{x}=\frac{1}{C_{\text {theo }}}
$$

and

$$
\delta_{i}=\frac{\lambda_{i}}{1-\lambda_{i} h_{x}}
$$

At a macroscopic level, the weaving section is seen as the superposition of two merges and two diverges. The global model will be elaborated by (i) specifying traffic behaviors for those local units and by (ii) sketching out its operation when introducing feedbacks between the different components of the overall model. To synthesize the operation of the overall model, Figure 2 is a scheme presenting the feedbacks between the different components which will be presented later in the chapter. Two mechanisms can dictate the operation of the weaving section when lane $i$ is congested and lane $j$ is in free-flow:

- Firstly - the weaving vehicles exiting lane $i$ can anticipate their lane change and reduce their speed inside the anticipation zone. If the number of weaving vehicles coming from lane $i$ is high enough, the operation of the weaving section is dictated by the model presented in subsection 2.4, see Block 1 in Figure 2. This model gives an effective flow $q_{i}^{d}$;

- Secondly - the weaving vehicles coming from lane $j$ and merging on lane $i$ can also degrade the traffic conditions in lane 1. In this case, the model presented in subsection 2.2 describes the operation of the weaving section, see Block 2 in Figure 2. This model gives an effective flow $q_{i}^{m}$.

At the end, the output of the model is the effective flow $q_{i}$ on the congested lane $i$ which is the minimum between $q_{i}^{d}$ and $q_{i}^{m}$. The model is strictly symmetric when considering a situation for which only lane $j$ is saturated. When both incoming roads of the weaving section are congested, we assume moreover that the effective flows fairly share the available downstream capacity. 


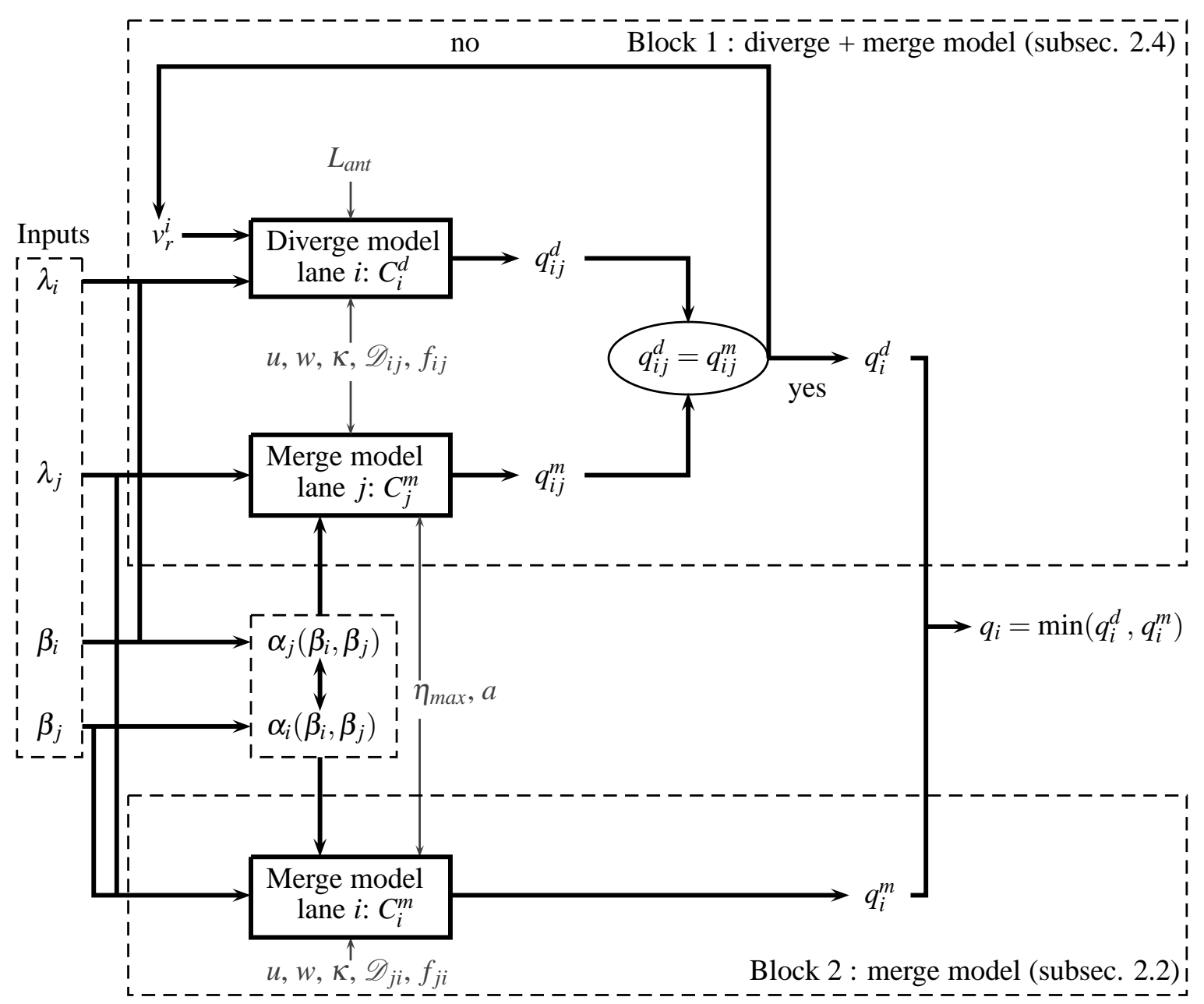

Figure 2: Feedbacks between the different components of the global model

Table 1 presents all the variables included in the model and their definitions. 


\begin{tabular}{|c|c|c|}
\hline Notation & Definition & Classification \\
\hline$\beta_{i}$ & Percentage of weaving flow from lane $i(\%)$ & Input variable \\
\hline$\lambda_{i}$ & Demand on lane $i(\mathrm{veh} / \mathrm{s})$ & Input variable \\
\hline$q_{i}$ & Effective flow on lane $i(\mathrm{veh} / \mathrm{s})$ & Output variable \\
\hline$q_{i}^{m}$ & Effective flow on lane $i$ given by the merge model (veh/s) & Aux. variable \\
\hline$q_{i}^{d}$ & Effective flow on lane $i$ given by the diverge model (veh/s) & Aux. variable \\
\hline$C_{\text {theo }}$ & Theoretical capacity (veh/s) & Aux. variable \\
\hline$C^{m}$ & Merge effective capacity (veh/s) & Aux. variable \\
\hline$C^{d}$ & Diverge effective capacity (veh/s) & Aux. variable \\
\hline$h_{x}$ & Safety time headway (s) & Aux. variable \\
\hline$u$ & Free flow speed $(\mathrm{m} / \mathrm{s})$ & Parameter $(\mathrm{m})$ \\
\hline$w$ & Wave speed in congestion $(\mathrm{m} / \mathrm{s})$ & Parameter $(\mathrm{m})$ \\
\hline$\kappa$ & Jam density (veh/m) & Parameter $(\mathrm{m})$ \\
\hline$a$ & Acceleration $\left(\mathrm{m} / \mathrm{s}^{2}\right)$ & Parameter (c) \\
\hline$\eta_{\max }$ & Maximum relaxation & Parameter (c) \\
\hline$L_{\text {ant }}$ & Length of the anticipation zone $(\mathrm{m})$ & Parameter (c) \\
\hline$\tau$ & Disturbance time (s) & Aux. variable \\
\hline$\alpha_{i}$ & Merge-ratio (merge model on lane $i$ ) & Aux. variable \\
\hline$\mu$ & Proportionality between $\alpha_{1}$ and $\alpha_{2}$ & Aux. variable \\
\hline$v_{r}$ & Slowdown speed $(\mathrm{m} / \mathrm{s})$ & Aux. variable \\
\hline$v_{0}$ & Inserting speed $(\mathrm{m} / \mathrm{s})$ & Aux. variable \\
\hline $\mathscr{D}_{i j}$ & Distribution of the lane changing positions from lane $i$ to lane $j$ & Aux. variable \\
\hline$p_{i j}$ & $\mathscr{D}_{i j}$ parameters & Aux. variable \\
\hline$s_{x}$ & Inserting positions standard deviation (m) & Aux. variable \\
\hline$s_{h}$ & Headways standard deviation (s) & Aux. variable \\
\hline$s$ & Standard deviation of the inserting process (s) & Aux. variable \\
\hline
\end{tabular}

Table 1 : Notations, definitions and classification: $(m)$ et (c) stand for the parameters that need to be measured and the parameters that need to be calibrated respectively 


\subsection{Merge component}

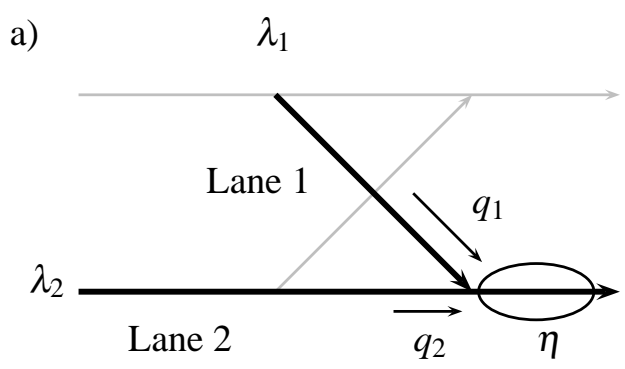

b)

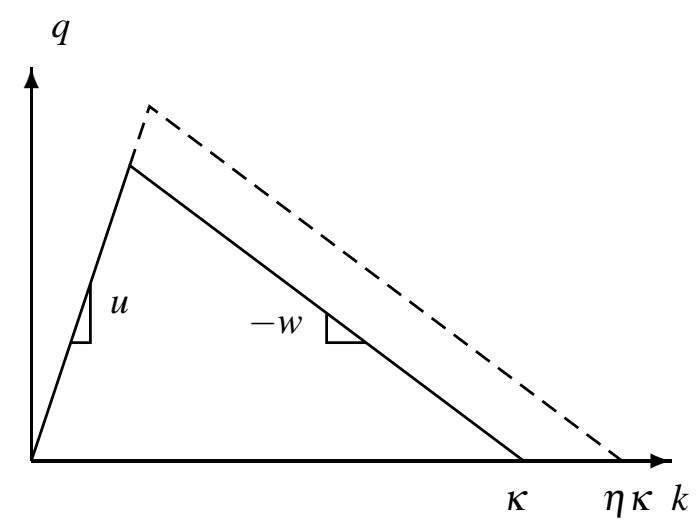

Figure 3 : a) Merge component and b) triangular fundamental diagram with the relaxation

The merge component is a refinement of the merge model originally presented in (Leclercq et al., 2011) and referred to as LL-model in the remainder of this paper. Let us consider the simple merge depicted in Figure 3a. $\lambda_{2}$ is the demand upstream of the main road (lane 2 in Figure 3a), while $\lambda_{1}$ is the demand upstream of the on-ramp (lane 1 in Figure 3a).

It is assumed in (Leclercq et al., 2011) that the demand on lane 2 is high enough to create congestion. The LL-model relies on the assumption that the lane-changers merge on lane 2 at a speed $v_{0}$ then accelerate at a constant acceleration rate $a$. According to the theory developed in (Laval and Daganzo, 2006), the accelerations create voids in the traffic stream that reduce the effective capacity of the merge. Leclercq et al. (2011) evaluate at a large time scale the number of vehicles between two successive insertions using the variational theory. The merging process reduces the effective capacity $C^{m}$ downstream of the merge to Eq.(5).

$$
C^{m}=w \kappa+w \eta \lambda_{1}\left(\frac{w+v_{0}}{a}-\frac{1}{a} \sqrt{\left(w+v_{0}\right)^{2}+\frac{2 a w}{\lambda_{1}}}+\frac{a s^{2} w^{2}}{2\left(\left(w+v_{0}\right)^{2}+\frac{2 a w}{\lambda_{1}}\right)^{3 / 2}}\right)
$$

Assuming that the inserting positions and the inserting times are independent, $s$, the standard deviation of their 
sum is $s=\sqrt{s_{h}^{2}+\frac{2 s_{x}^{2}}{w^{2}}}$ where $s_{h}$ and $s_{x}$ are the standard deviations of the headways distribution and the inserting positions distribution, respectively.

Leclercq et al. (2011) distinguish two situations whether lane 1 is free flowing or not. If lane 1 is in freeflow, it is assumed that the mergers instantaneously adapt the mean speed of the main road. This assumption leads to $v_{0}=\frac{w q_{2}}{w \kappa-q_{2}}$ and $C^{m}$ becomes a function of $q_{2}$. As $C^{m}$ is shared by $\lambda_{1}$ and $q_{2}$, one obtains Eq.6 and $q_{2}$ is numerically computed by solving this equation.

$$
C^{m}\left(q_{2}\right)=\lambda_{1}+q_{2}
$$

In case of queuing on both incoming links, the effective flows share the available downstream capacity according to a fixed merge ratio $\alpha$ (Daganzo, 1995). Although there is no general definition of the merge-ratio (Torné et al., 2014), in the present paper, $\alpha$ is the ratio between both incoming link capacities. The merge ratio holds thus $q_{1}=\alpha q_{2}$. As the on-ramp is saturated, the merging speed is simply $v_{0}=\frac{w q_{1}}{w \kappa-q_{1}}$ and $C^{m}$ becomes a function of $q_{1}$. The effective flows are computed solving Eq. 7 .

$$
C^{m}\left(q_{1}\right)=\left(1+\frac{1}{\alpha}\right) q_{1}
$$

The relaxation phenomenon takes place when vehicles involved in a lane change accept shorter spacings then gradually adapt their speed to reach their equilibrium spacings. The merging vehicles in relaxation induce non-equilibrium traffic states that increase the effective flow. The LL-model does not take into account this phenomenon and, as a consequence, it underestimates the effective capacity of the merge. To address this issue we add a dynamic relaxation factor $\eta$ to the fundamental diagram downstream of the merge, see Figure $3 b$. If the number of vehicles coming from the unsaturated lane is low, the relaxation process affects only a couple of vehicles and therefore has little impact at a macroscopic level. We assume that $\eta$ is a linear increasing function of $\lambda_{1}$ which is the demand coming from the on-ramp. The relaxation function is given by a single parameter $\eta_{\max }$ corresponding to the maximum allowed relaxation parameter. We have:

$$
\eta(\lambda)= \begin{cases}1 & \text { if } \lambda \leq \lambda_{1}^{c} \\ \frac{\eta_{\max }-1}{\alpha q_{\alpha}-\lambda_{1}^{c}}\left(\lambda-\lambda_{1}^{c}\right)+1 & \text { elseif }\end{cases}
$$

Where $q_{\alpha}$ is the flow on the main road when both incoming links of the merge are saturated.

Moreover Leclercq et al. (2011) expressed $s_{x}$ simply as a function of the on-ramp length. They suppose therefore that the merging behavior depends on the road configuration but not on the prevailing traffic conditions on the target lane. However, recent empirical studies proved that either the road configuration or the traffic 
conditions influence the merging behavior (Daamen et al., 2010; Marczak et al., 2013). We address this issue by adjusting the standard deviation according to the mean speed on the target lane $v_{2}$. The positions of the lane changes, $X$, are assumed to follow a theoretical distribution $\mathscr{D}_{12}$ that depends on a vector of parameters $p_{12}$ :

$$
X \sim \mathscr{D}_{12}\left(p_{12}\right)
$$

Where $p_{12}$ is a function of the effective flow on the target lane, $p_{12}=f_{12}\left(q_{2}\right)$. $\mathscr{D}_{12}$ and $f_{12}$ will be adjusted from empirical considerations later in the paper.

$s_{h}$ is a parameter of the original LL-model. To reduce the number of parameters of our model, we propose to adjust endogenously $s_{h}$ as a function of the demand on the on-ramp. We have assumed that the headways follow a shifted exponential distribution. $s_{h}$ can be directly derived from Eq.(2):

$$
s_{h}\left(\lambda_{1}\right)=\frac{1}{\delta_{12}}=\frac{1}{\lambda_{1}}-\frac{1}{C_{\text {theo }}}
$$

Finally we have the following expression of the effective capacity:

$$
C^{m}=w \eta \kappa+w \eta \kappa \lambda_{12}\left(\frac{w+v_{0}}{a}-\frac{1}{a} \sqrt{\left(w+v_{0}\right)^{2}+\frac{2 a w}{\lambda_{1}}}+\frac{a s\left(\lambda_{1}\right)^{2} w^{2}}{2\left(\left(w+v_{0}\right)^{2}+\frac{2 a w}{\lambda_{1}}\right)^{3 / 2}}\right)
$$

With

$$
s=\sqrt{\left(\frac{1}{\lambda_{1}}-\frac{1}{C_{\text {theo }}}\right)^{2}+\frac{2 \operatorname{var}(X)}{w^{2}}}
$$

It is worth noting that the shape of the headways distribution is not important only the standard deviation is. We have performed extensive numerical analysis for admissible ranges of parameters for $q_{2}$ between 0 and $C_{\text {theo }}$. It appears that the upper and lower bounds of Eqs. (6) and (7) are positive and negative, respectively. As Eqs.(6) and (7) are continuous function of $q_{2}$, they admit at least one solution. 


\subsection{Diverge component}

a)

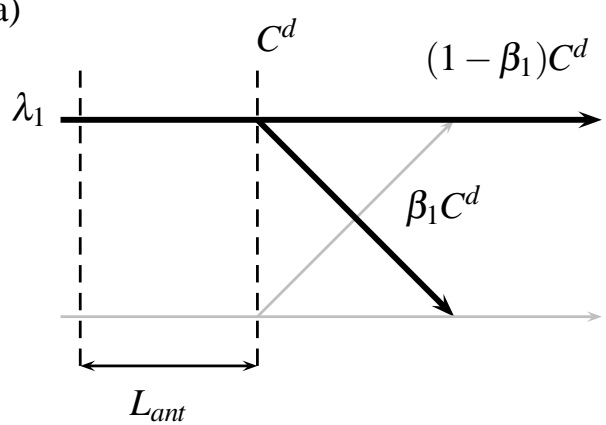

b)

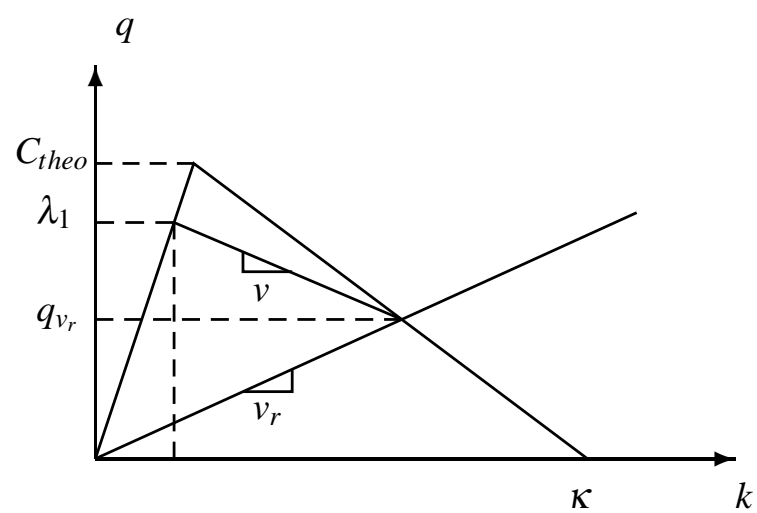

Figure $4:$ a) Sketch of the theoretical diverging junction and b) illustration of the effect of a moving bottleneck driving at $v_{r}$

The weaving model presented in Figure 2 introduces a diverge component. This paragraph presents an analytical expression of the capacity of a diverging junction with one lane on the main road and one lane on the off-ramp, see Figure 4a. Laval (2009) developed a framework to analytically estimate the capacity reduction caused by trucks forced to slow down with infinite deceleration at an uphill segment on a multilane freeway. The trucks are assumed to drive at a speed $v_{r}$, which is lower than $u$, inside the uphill segment. Assuming that the exiting vehicles anticipate their maneuver inside a $L_{a n t}$-long anticipation zone and slow down at a speed $v_{r}$ this framework was further elaborated in the case of a simple diverging section with bounded accelerations (Marczak and Buisson, 2014), see Figure Figure 4a. The headways between two successive slow moving vehicles are moreover assumed to be constant in (Marczak and Buisson, 2014) while there are random in (Laval, 2009).

In accordance with Newell's kinematic wave theory, the slow moving vehicles are considered as moving bottlenecks. If $\lambda_{1}$ is high enough, the moving bottlenecks introduce shockwaves that propagate upstream at a wave speed $v$ and reduce the effective flow at the diverging point, see Figure $4 \mathrm{~b}$. The expression given in (Marczak and Buisson, 2014) underestimates the capacity of the diverging junction for lower rates of slow moving vehicles. We consider therefore the analytical model presented in (Laval, 2009). Considering the 
renewal theory, Laval (2009) expresses the distribution of the headways between two successive slow moving vehicles at the beginning of the anticipation zone. The headways follow an exponential distribution whose density function is:

$$
f_{H}(h)= \begin{cases}l_{1} e^{-l_{1} h} & \text { if } h \leq \tau\left(v_{r}\right) \\ e^{\tau\left(v_{r}\right)\left(l_{0}-l_{1}\right)} l_{0} e^{-l_{0} h} & \text { if } h>\tau\left(v_{r}\right)\end{cases}
$$

With

$$
\begin{aligned}
& \tau\left(v_{r}\right)=L_{a n t} \frac{v_{r}+w}{v_{r} w} \\
& l_{0}=\beta_{1} \frac{u w \kappa}{u+w} \quad, \quad l_{1}=\beta_{1} \frac{v_{r} w \kappa}{v_{r}+w}
\end{aligned}
$$

Where $\tau$ is the disturbance time introduced by a slow moving vehicle and $l_{0}$ and $l_{1}$ are the mean slow moving vehicles arrival rate at the beginning of the anticipation zone when the flow at this point is $C_{t h e o}$ or $q_{v_{r}}$, respectively, see Figure 4b.

Finally, the effective capacity of the diverge, $C^{d}$ is simply given by

$$
C^{d}=\frac{1}{\beta_{1} \bar{H}}
$$

Where $\bar{H}$ is the mean headway between two successive exiting vehicles. $\bar{H}$ can be immediately derived from Eq.(13). Note that randomly drawn lane changing positions do not influence the capacity of the diverge. Indeed, we assume that all the exiting vehicles drive at $v_{r}$. The drop in capacity occurs therefore at the beginning of the anticipation zone independently of the lane changing positions.

\subsection{Combining one upstream diverge with the associated downstream merge}

From the two previous steps we have the capacity of a merge $C^{m}$ and the capacity of a diverge $C^{d}$. The next step is to combine a diverge on lane 1 with a merge on lane 2, see Figure 5a. The developments presented in this section will be identical when combining one merge on lane 1 and one diverge on lane 2 . The main road of the merge is assumed to be lane 2 . We assume moreover in this subsection that $\lambda_{2}$ is low enough to prevent congestion on lane 2 because the situation for which lane 2 is saturated can be analytically solved with the merge model presented in subsection 2.2. The lane changing vehicles coming from lane 1 temporarily drive at $v_{r}$ inside the anticipation zone. The demand on lane 1 is high enough to initiate shockwaves that reduce the effective flow at the diverging point. 
a)

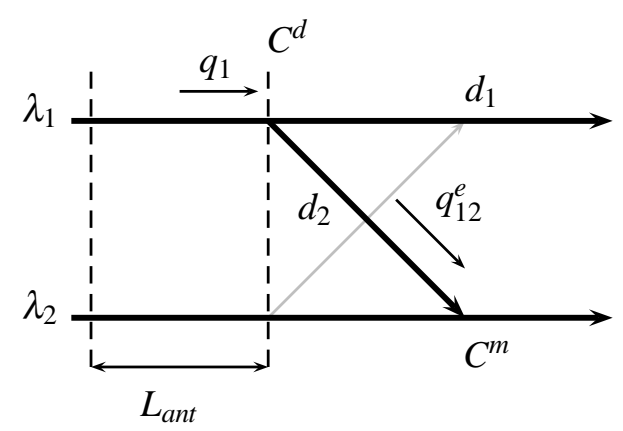

b)

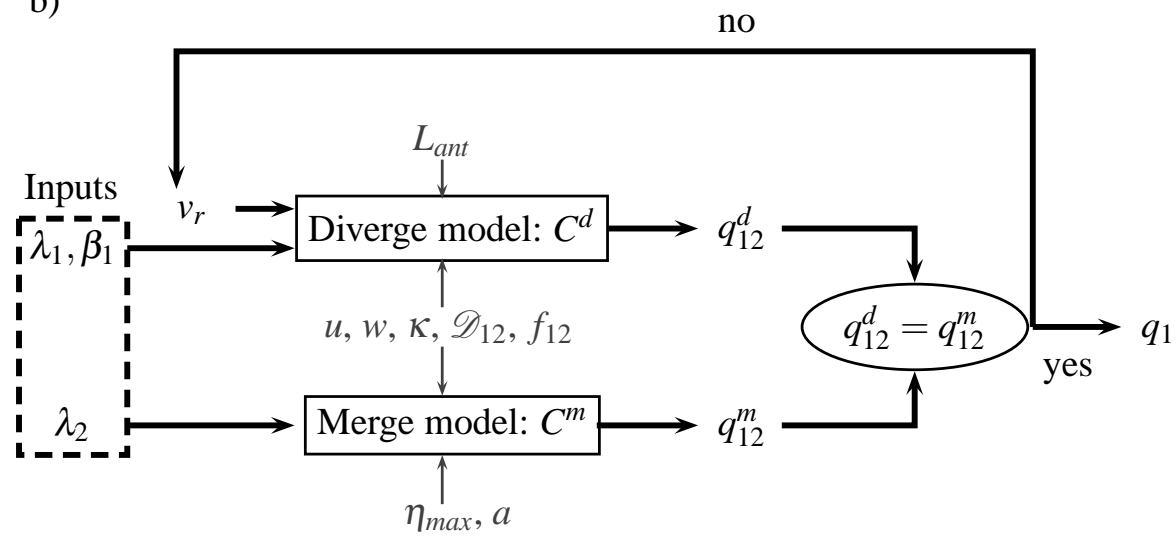

Figure 5 : a) Combining one merge and one diverge and b) focus on block 1 of Figure 2

There are two links, respectively denoted $d_{1}$ and $d_{2}$, downstream of the diverge on lane 1, see Figure 5a. No capacity reduction occurs downstream of $d_{1}$. Therefore, the effective capacity of $d_{1}$ equals $C_{\text {theo }}$. Because of the merging process, the capacity on $d_{2}$ is reduced to $C^{m}$, which is the capacity given by the merge model for a fixed $\lambda_{2}$. The merge model estimates also the effective lane changing flow $q_{12}^{m}$ coming from lane 2, see Figure 5b. But for a given $v_{r}$, the diverge model proposes another lane changing flow $q_{12}^{d}$. As $u, w, \kappa, L_{a n t}, \eta_{\max }$ and $a$ are fixed, we propose to adjust $v_{r}$ to ensure equality between $q_{12}^{m}$ and $q_{12}^{d} . v_{r}$ is found so that the following equation is satisfied:

$$
\lambda_{2}+\beta_{1} C^{d}\left(v_{r}\right)=C^{m}\left(v_{r}, s^{d}\left(v_{r}\right)\right)
$$

Once again, assuming that the headways between two successive lane changes and the lane changing positions are independent, one can write:

$$
s^{d}\left(v_{r}\right)=\sqrt{s_{H}^{2}+\frac{2 \operatorname{var}(X)}{w^{2}}}
$$

Where $s_{H}$ is the standard deviation of the headways distribution given by Eq.(13).

$v_{r}$ was a parameter of the diverge model presented in subsection 2.3. When combining a diverge and a 
merge $v_{r}$ is no longer a parameter of the model, but $v_{r}$ is adjusted endogenously as a function of $\lambda_{2}$. This is the feedback between the different models. One again we have performed extensive numerical resolutions for an admissible range of parameters. It appears that Eq.(16) always admits a solution. Interestingly, Figure 6 shows that $v_{r}$ is a decreasing function of $\lambda_{2}$. When the flow is higher on lane 2 , the drivers coming from lane 1 anticipate more their lane changes because the offered gaps on lane 2 are smaller and the lane changes are therefore more difficult.

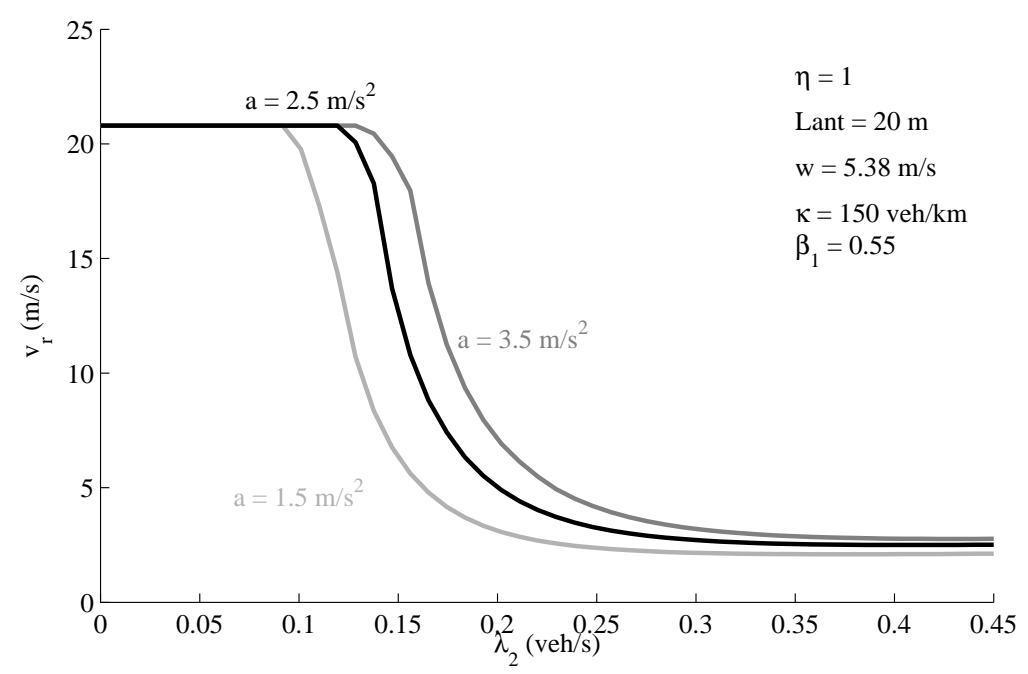

Figure 6 : Evolution of $v_{r}$ as a function of $\lambda_{2}$ for different accelerations

\subsection{The overall model}

The goal now is to describe the complete analytical model for the weaving section. At a macroscopic level, the weaving section is represented as the superposition of two merges and two diverges.

When both incoming roads are congested, we assume moreover that the effective flows fairly share the available downstream capacity according to a fixed priority ratio which is equal to 1 and independent of the percentages of weaving flows $\beta_{1}$ and $\beta_{2}$. As a consequence the merge-ratios $\alpha_{1}$ and $\alpha_{2}$ are dynamic and dependent on $\beta_{1}$ and $\beta_{2}$. This is a strong assumption because usually $\alpha$ is supposed independent of the upstream demand.

Bar-Gera and Ahn (2010) show empirically that the merge-ratios for independent merges can be reasonably estimated by the ratios between the number of lanes on the main road and the number of lanes on the onramp. The findings of this study indicate that the merge-ratios depend on the geometrical configuration of the merge area and consequently on the infrastructure supply. But the authors pinpoint some residual differences between merge-ratios and lane-ratios. They suggest therefore that other factors influence the merge-ratios. More recently, Reina and Ahn (2014) developed a formulation of merge-ratios using lane flow distribution (LFD). The authors showed that the proposed LFD-based model can capture variation in merge-ratios with 
respect to the traffic conditions. Chevallier and Leclercq (2007) considered in simulation a dynamic priority sharing ratio to reproduce the drivers' aggressiveness. Moreover these studies are for independent merges and not for weaving sections. Note that this assumption on $\alpha_{1}$ and $\alpha_{2}$ can be relaxed without changing the model structure when further and specific data for weavings will be collected.

In case of continuous queuing on both incoming links, one can guarantee the consistency of the effective flow estimated by both merge models by writing:

$$
\left\{\begin{array}{l}
q_{\alpha_{1}}=\frac{\alpha_{2} q_{\alpha_{2}}}{\beta_{1}} \\
q_{\alpha_{2}}=\frac{\alpha_{1} q_{\alpha_{1}}}{\beta_{2}}
\end{array}\right.
$$

Interestingly, if Eq. (18)(a) and (b) are verified they yield the following simple condition:

$$
\alpha_{1} \alpha_{2}=\beta_{1} \beta_{2}
$$

One can assume moreover that $\alpha_{1}$ and $\alpha_{2}$ are proportional and that there is a constant $\mu$ such that :

$$
\alpha_{2}=\mu \alpha_{1}
$$

One can write therefore

$$
\alpha_{1}=\sqrt{\frac{\beta_{1} \beta_{2}}{\mu}}, \quad \alpha_{2}=\sqrt{\mu \beta_{1} \beta_{2}}
$$

The remaining question to adjust endogenously $\alpha_{1}$ and $\alpha_{2}$ is the estimation of $\mu$. We also assume that the effective flows on lane 1 and lane 2 compete to fairly share the downstream capacity on a one-to-one basis. One should have consequently:

$$
\min \left(q_{\alpha_{1}}, \frac{\alpha_{2} q_{\alpha_{2}}}{\beta_{1}}\right)=\min \left(q_{\alpha_{2}}, \frac{\alpha_{1} q_{\alpha_{1}}}{\beta_{2}}\right)
$$

One can easily estimate $\mu$ solving Eq.(22).

Previous studies using micro-simulation to estimate the capacity of a weaving section, pinpointed that the length of the weaving section is a key factor influencing its capacity (Dijker and Schuurman, 2003; Calvert and Minderhoud, 2012). Our model does not include explicitly the length of the weaving section as a parameter. It is, however, important to stress that the length is implicitly included in the distribution of the lane changing positions. A longer weaving section may indeed introduce different lane changing behaviors and therefore a different distribution of the lane changing positions. As the standard deviation of this distribution is a parameter 
of the model, the length of the weaving section is implicitly taken into account in the analytical expression of the capacity.

\section{Sensitivity analysis}

A sensitivity analysis is now performed to determine the contribution of the different parameters to the capacity drop. Let us denote $C$ the effective capacity of the weaving section. We define $C$ as the effective flow passing the weaving section when both lane 1 and lane 2 are saturated. As we assumed that the main road and the auxiliary lane obey the same fundamental diagram, we have simply $C=2 C_{\text {theo }}$. The sensitivity to the parameters and the relative capacity drop are quantified with $c$ the complement of the ratio between the effective capacity and the theoretical capacity (Leclercq et al., 2011). We focus the sensitivity analysis on the acceleration rate $a$, the maximum relaxation factor $\eta_{\max }$ and the length of the anticipation zone $L_{a n t}$. The results of the sensitivity analysis are presented in Figure 7. The dotted lines represent $c$ for the situation with randomly drawn headways between two successive lane changes and a fixed lane changing position. The continuous lines represent $c$ for randomly drawn headways and lane changing positions. 
a) Sensitivity to a

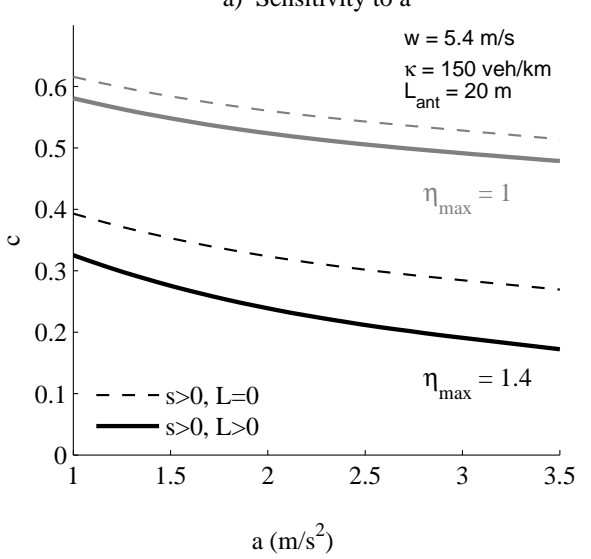

c) Sensitivity to Lant

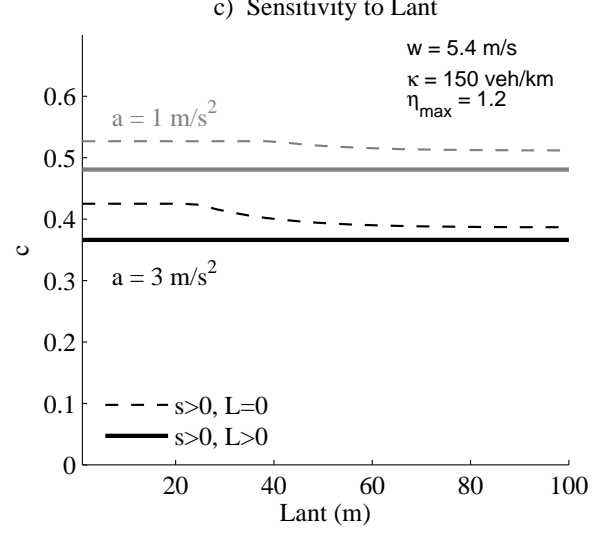

b) Sensitivity to $\eta_{\max }$

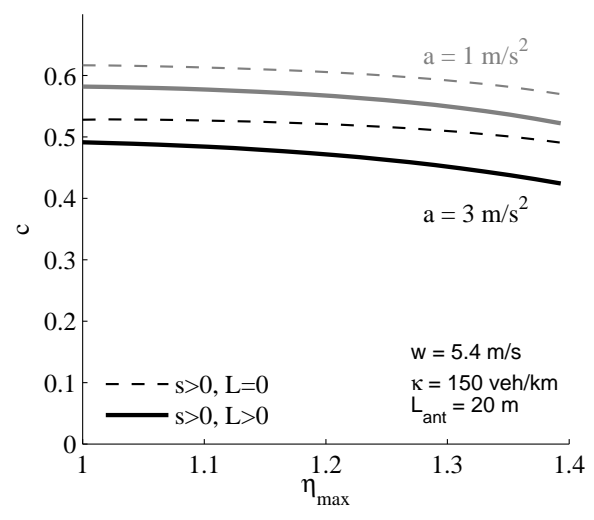

d) Sensitivity to $\beta_{1}$ and $\beta_{2}$

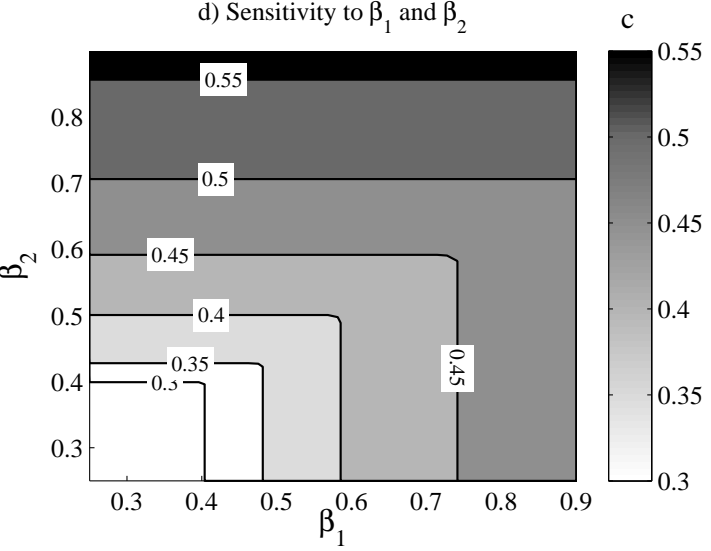

Figure 7 : Sensitivity analysis: influence on c, the relative capacity drop of the weaving section, of a) the acceleration rate $a, \mathrm{~b}$ ) the maximum relaxation factor $\left.\eta_{\max }, \mathrm{c}\right)$ the length of the anticipation zone $L_{\text {ant }}$, and d) the percentages of weaving flows $\beta_{1}$ and $\beta_{2}$. We have chosen $\beta_{1}=0.55$ and $\beta_{2}=0.59$ in the first three cases

while we have fixed $a=2 \mathrm{~m} / \mathrm{s}^{2}, \eta_{\max }=1.2, L_{\text {ant }}=20 \mathrm{~m}, w=5.4 \mathrm{~m} / \mathrm{s}$ and $\kappa=0.15 \mathrm{veh} / \mathrm{m}$ to study the sensibility to $\beta_{1}$ and $\beta_{2}$

Figure $7 \mathrm{a}$ shows the influence of $a$ on the relative capacity drop for different values of $\eta_{\max }$. Figure $7 \mathrm{~b}$ presents the influence of $\eta_{\max }$ on the capacity drop for different values of $a$. It appears that increasing $a$ or $\eta_{\max }$ increases the effective capacity and reduces consequently $c$. When $a$ is high, the voids created by the acceleration process in the traffic stream are smaller. The effective flow is therefore higher. $\eta_{\max }$ is a dilatation factor of the fundamental diagram. It increases the effective flow crossing the weaving section. Figure $7 \mathrm{c}$ focuses on $L_{a n t}$ for different values of $a$. $L_{a n t}$ hardly influences the effective capacity. It appears moreover that the capacity drop is lower when the lane changing positions are randomly drawn. The effective capacity of the weaving section is therefore higher. Eq. (12) proves that one increases the standard deviation when considering randomly drawn headways and lane changing positions. Leclercq et al. (2011) show that the standard deviation reduces the relative capacity drop.

The capacity of a weaving section varies with respect to the percentages of weaving flows (Lertworawanich and Elefteriadou, 2003). We analyze therefore the sensitivity of the model to $\beta_{1}$ and $\beta_{2}$. Figure $7 \mathrm{~d}$ is an illustration of the $\left(\beta_{1}, \beta_{2}\right)$ plane which is split in different regions according to the relative capacity drop $c$. 
These regions are bounded by isolines of $c$. First of all, one can observe that $c$ is not symmetrical with respect to $\beta_{1}$ and $\beta_{2}$. The standard deviation of the lane changing positions is a parameter of the model. The position distribution of the lane changes towards the auxiliary lane differs from that towards the main road because their respective parameters are not the same, see Figure 9. One observes therefore asymmetrical effects on $c$ because those distributions, and hence the standard deviations, are different. For the lower values of $\beta_{1}$ and $\beta_{2}, c$ is less than 5\%. $c$ increases with increasing $\beta_{1}$ and $\beta_{2}$ : the higher the number of weaving vehicles, the higher the drop in capacity. For the higher values of $\beta_{1}$ and $\beta_{2}$, less than $45 \%$ of the theoretical capacity are used.

\section{Model verification and validation}

\subsection{Comparison with empirical observations}

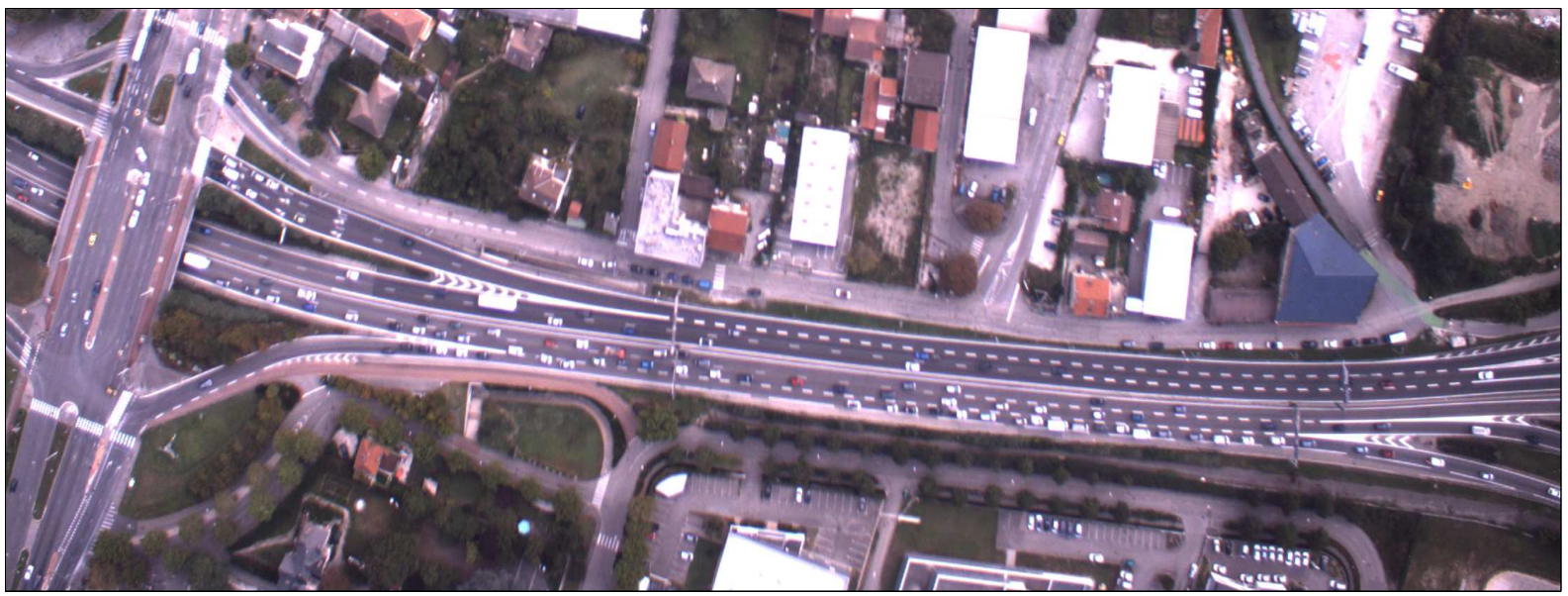

Figure 8 : Data collection site in Grenoble. The traffic moves from left to right.

The weaving study site is shown in Figure 8 (MOCoPo, 2011). It is at the junction between RN87 and A480 in the south east part of Grenoble. The site is a weaving section with a two-lane and single-lane entry legs and three single-lane exit legs. The maximum allowed-speed on the main road is $70 \mathrm{~km} / \mathrm{h}(\approx 20 \mathrm{~m} / \mathrm{s})$. The block line between the main road and the auxiliary lane has a length of $250 \mathrm{~m}$. The video recordings have been collected using a high-resolution video camera mounted underneath a helicopter. The images have been stabilized and the trajectories have been extracted using a dedicated software (Knoppers et al., 2012). As in (Marczak et al., 2014), we consider in this paper two one-hour samples respectively recorded on Thursday, September 15th 2011 and Friday, September 16th 2011. The traffic on the studied weaving section is local. The drivers know the specific geometrical configuration of the weaving section and choose their lane as soon as possible to reach their destination. One observes therefore very few lane changes from the left lane to the right lane of the freeway and vice versa. The lane changes occur mainly between the right lane of the freeway and the auxiliary lane. The studied weaving section may be thus assimilated to the theoretical weaving section 
depicted in subsection 2.1
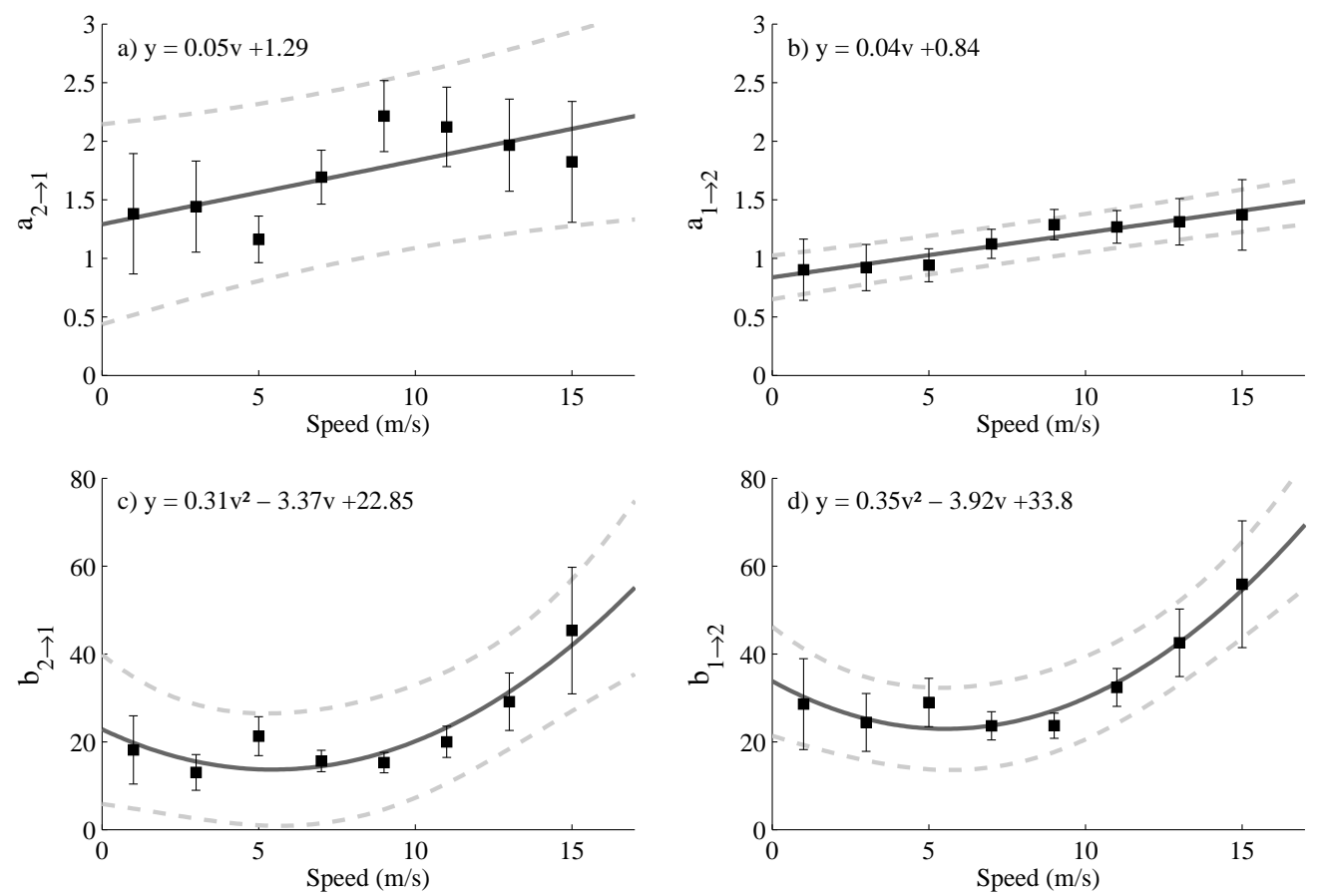

Figure 9: Maximum Likelihood Estimations for the parameters of the adjusted distributions

First of all, we fit empirically the distribution $\mathscr{D}$ of the lane changing positions. The data have been aggregated using the lane changing speed. We assume that this speed gives an accurate indication of the prevailing traffic conditions on the target lane. The data have been fitted with different theoretical distributions inside each speed class. It appears that the Gamma distribution fits the best the empirical observations. Figure 9 presents the Maximum Likelihood Estimations for the parameters of the adjusted distribution. $a_{i \rightarrow j}$ and $b_{i \rightarrow j}$ are the shape parameter and the scale parameter, respectively, of the Gamma distribution. We also represent the confidence intervals for $a_{i \rightarrow j}$ and $b_{i \rightarrow j}$ at a 5\% significance level. Then $a_{i \rightarrow j}$ and $b_{i \rightarrow j}$ have been adjusted as functions of the speed with the simplest polynomial. The solid lines in Figure 9 are the adjusted polynomials.

Microscopic trajectory data allow us to trace each vehicle from its origin at the beginning of the weaving section to its destination at the end of the weaving section. We can therefore have an accurate estimation of $\beta_{1}$ and $\beta_{2}$. However we do not have enough data for a given couple of $\beta_{1}$ and $\beta_{2}$. Moreover, we cannot observe all the possible capacities because of the numerous combinations of percentages of weaving vehicles. To address this issue, we extracted from the video recordings some periods for which the weaving section is either free flowing or an an active bottleneck. The bottleneck is located at the start of the block line between the main road and the auxiliary lane. When the bottleneck is active, the congestion moves upstream $(i)$ only on the rightmost lane on the main road or (ii) on the auxiliary lane and the rightmost lane. We have taken only the situations in congestion for which the mean speed is lower than $40 \mathrm{~km} / \mathrm{h}(11 \mathrm{~m} / \mathrm{s})$. We have computed from the trajectories the time instants at which the vehicles pass through a fictive detector located at the start of the 
block line. Then the data have been aggregated over a two-minute time interval to obtain homogeneous traffic state and to estimate the effective flows and $\beta_{1}$ and $\beta_{2}$. Finally we have determined respectively the maximum and minimum observed couples of $\beta_{1}$ and $\beta_{2}$.

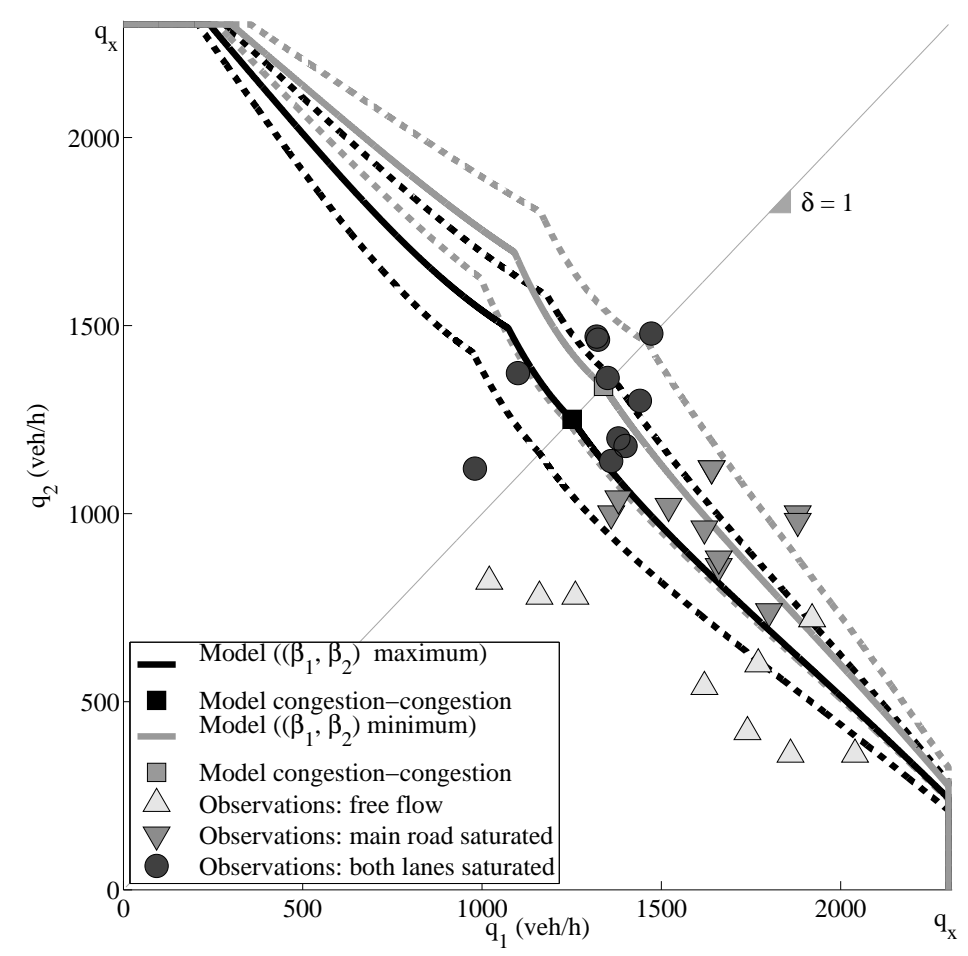

Figure 10 : Comparison between empirical observations and the analytical capacity curves. The observations have been aggregated over a two-minute time interval.

Figure 10 presents the comparison between the empirical observations and the theoretical capacity curves which are the continuous lines. We fixed $\eta_{\max }=1.13$ and $a=2.5 \mathrm{~m} / \mathrm{s}^{2}$. The fundamental diagram has been calibrated on each lane of the studied weaving section during congestion. We have $u=20 \mathrm{~m} / \mathrm{s}, w=5.38 \mathrm{~m} / \mathrm{s}$ and $\kappa=0.15 \mathrm{veh} / \mathrm{m}$. The lower measured percentages of weaving flows is $\beta_{1}=0.55$ and $\beta_{2}=0.59$ while the higher couple is $\beta_{1}=0.69$ and $\beta_{2}=0.67$. We estimate that $\beta_{1}$ and $\beta_{2}$ have been measured with a precision of $15 \%$. For each couple of $\beta_{1}$ and $\beta_{2}$ we construct therefore a lower bound of the capacity curve with $0.85 \beta_{1}$ and $0.85 \beta_{2}$, and an upper bound with $1.15 \beta_{1}$ and $1.15 \beta_{2}$. The bounds of the capacity curves are the dashed lines.

The empirical observations fall in the regions of the model predictions. One can first of all note that the observations in free-flow are below the capacity curves. The observations in congestion are between the extreme capacity curves. When the rightmost lane on the main road and the auxiliary lane are congested, the effective flows on each lane share fairly the effective capacity of the weaving section. The observations are indeed distributed near the identity function. These empirical findings are coherent with the model theory. 


\subsection{Comparison with simulation results}

A second option for questioning our analytical model is to compare it with simulation results. The simulated weaving vehicles will temporarily consider their leader on the adjacent lane if the mean speed on the actual lane is higher than the mean speed on the adjacent lane. The leader on the adjacent lane is the closest vehicle driving in front of the considered weaving vehicle. The car following rule is an extension to Newell's car-following model (Newell, 2002) with a relaxation term (Laval and Leclercq, 2007) and bounded acceleration (Leclercq, 2007). We redirect our readers to (Leclercq et al., 2007) for a more precise description of the car-following model. The lane changing model simply expresses the probability of changing lane as a function of the longitudinal position. The lane changing positions follow a Gamma distribution. The parameters of the distribution are adjusted according to the results presented in Figure 9. The demands upstream of the weaving section are constant and the headways upstream of the weaving section are assumed to follow a shifted exponential distribution whose density function is given by Eq.(2). We run 1500 replications. For each replication, we simulate 15 minutes of data. Then, we construct the curve of cumulative vehicles count (CVC) $1000 \mathrm{~m}$ downstream of the weaving section according to the origin of the vehicles. Finally, the effective capacity is the long-term average, measured with the slope of the CVC during the last 6 minutes of the simulation.
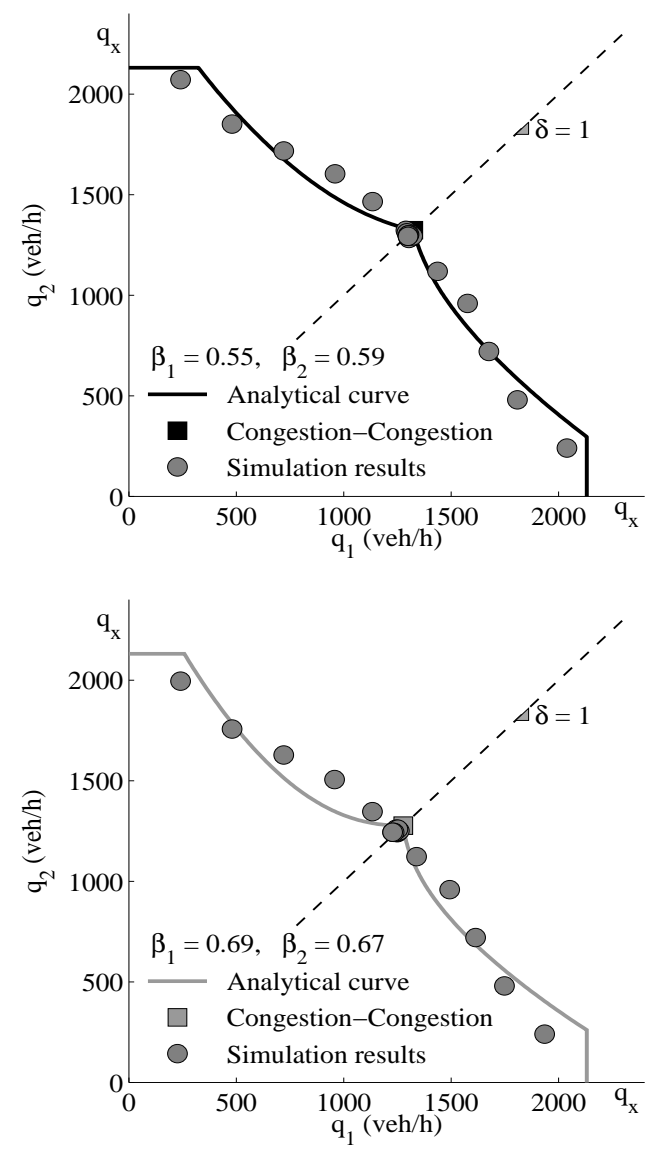

Figure 11 : Comparison between simulation results and the analytical capacity curves 
Figure 11 presents the comparison between the simulation results and the theoretical capacity curves for the extreme couples of $\beta_{1}$ and $\beta_{2}$. The simulation results and the analytical curves are in high accordance.

\section{Conclusions}

This paper presents a parsimonious macroscopic model for a freeway weaving section with an allocation scheme similar to the Newell Daganzo model and an endogenous expression of the capacity drop. The paper proposes an explicit formulation of the relation between microscopic interactions related to the weaving activity and their impact on the capacity at a macroscopic level. It is postulated in our study that either the slowdowns or the accelerations of the weaving vehicles create voids in the traffic stream, and that these voids reduce the capacity of the weaving section. We simply consider a theoretical weaving section as the superposition of two merges and two diverges. We assume therefore that the operation of the weaving section is dictated either by the operation of a single merge or by the operation of a single diverge. A parsimonious formulation of the capacity that depends only on six parameters is proposed. The acceleration and the relaxation parameter strongly influence the effective capacity, whereas the length of the anticipation zone hardly influences the capacity. The analytical expression of the capacity accords well with empirical observations and simulation results.

The work presented in this paper assumed some simplifying assumptions. The theory was developed for a very simple weaving section with one lane on the main road and one auxiliary lane. The traffic upstream of the weaving section is consequently strictly FIFO. The proposed model has to be further developed for other weaving configurations with more lanes on the main road. The merge model should specifically be generalized for configurations with more lanes on the main road. Extensions to multi-lanes freeways have been discussed in (Leclercq et al., 2011). But it is assumed that the capacity drop occurs (i) only on the shoulder lane while the other lanes are free flowing or (ii) identically on all downstream lanes. The model gives a bound of the capacity drop but it cannot reproduce properly lane flow distributions because it does not consider the discretionary lane changes that may reduce that capacity on the main road (Cassidy and Rudjanakanoknad, 2005). Research has to continue to integrate explicitly the discretionary lane changes in the analytical expression of a multilanes merge capacity. Similarly, we have considered a diverge with one lane of the main road. The traffic is consequently strictly FIFO. More research is needed to estimate the effective capacity of a non-FIFO diverge with more lanes on the main road. Then the enhanced merge and diverge models should be aggregated in order to build a generalized model for weaving sections.

The proposed model has been compared to a single dataset. Future research should be performed to collect field data on different weaving sections and compare the capacities predicted by the model to field-measured capacities. We have made a strong assumption on the priority ratio when both incoming roads are congested 
assuming that it equals 1 , independently of the percentages of weaving flows. This is in accordance to what was observed on the studied weaving section but must be compared to reality in more cases. Future empirical researches should estimate how the weaving ratio is linked to the percentages of weaving flows. As in (Sun and Elefteriadou, 2012) an instrumented vehicle-based experiment could be also designed to observe the drivers' action during the lane changes and have a deeper understanding of the anticipation behavior during those maneuvers.

\section{Acknowledgements}

Our thanks are to Anne-Christine Demanny (IFSTTAR/LICIT) for her careful reading of the paper and the anonymous referees for helping to greatly improve this article.

\section{References}

G. H. Bahm. A simple lane change model for microscopic traffic flow simulation in weaving sections. Transportation Letters: the International Journal of Transportation Research, 3(4):231-251, 2011.

H. Bar-Gera and S. Ahn. Empirical macroscopic evaluation of freeway merge-ratios. Transportation Research Part C: Emerging Technologies, 18(4):457-470, 2010.

S.C. Calvert and M.M. Minderhoud. Generic freeway weaving section capacity estimation through microsimulation. Transportation Research Board Annual Meeting, Paper 12-1026, 2012.

M.J. Cassidy and J. Rudjanakanoknad. Increasing the capacity of an isolated merge by metering its on-ramp. Transportation Research Part B: Methodological, 33(10):896-913, 2005.

M.J. Cassidy, A. Skabardonis, and A.D. May. Operation of major freeway weaving sections: recent empirical evidence. Transportation Research Record: Journal of the Transportation Research Board, 1225, pages 61-72, 1989.

E. Chevallier and L. Leclercq. A macroscopic theory for unsignalized intersections. Transportation Research Part B: Methodological, 41B(10):1139-1150, 2007.

W. Daamen, M. Loot, and S.P. Hoogendoorn. Empirical analysis of merging behavior at a freeway on-ramp. Transportation Research Record: Journal of the Transportation Research Board, 2188, pages 108-118, 2010.

C.F. Daganzo. The cell transmission model, Part II: network traffic. Transportation Research Part B: Methodological, 39(2):79-39, 1995. 
T. Dijker and H. Schuurman. The capacity of asymmetrical weaving sections. Transportation Research Board Annual Meeting, Paper 03-3066, 2003.

D. Gattuso, G. Musolino, and A. Tripodi. Experimental analysis of driver behaviour at unsignalized urban intersections. Recherche Transport Scurit, 88:205-224, 2005.

P. Hidas. Modelling vehicle interactions in microscopic simulation of merging and weaving. Transportation Research Part C: Emerging Technologies, 13(1):37-62, 2005.

P. Knoppers, H. Van Lint, and S.P. Hoogendoorn. Automatic stabilization of aerial traffic images. Transportation Research Board Annual Meeting, Paper 12-1674, 2012.

J.A. Laval. Effects of geometric design on freeway capacity: Impacts of truck lane restrictions. Transportation Research Part B: Methodological, 43(6):720-728, 2009.

J.A. Laval and C.F. Daganzo. Lane-changing in traffic streams. Transportation Research Part B: Methodological, 40B(3):251-264, 2006.

J.A. Laval and L. Leclercq. Microscopic modeling of the relaxation phenomenon using a macroscopic lanechanging model. Transportation Research Part B: Methodological, 42(6):511-522, 2007.

L. Leclercq. Bounded acceleration close to fixed and moving bottlenecks. Transportation Research Part B: Methodological, 41B(3):309-319, 2007.

L. Leclercq, N. Chiabaut, J.A. Laval, and C. Buisson. Relaxation phenomenon after changing lane: experimental validation with ngsim data. Transportation Research Record: Journal of the Transportation Research Board, 1999, pages 79-85, 2007.

L. Leclercq, J.A. Laval, and N. Chiabaut. Capacity drops at merges: an endogenous model. Transportation Research Part B: Methodological, 45(9):1302-1313, 2011.

J.H. Lee and M.J. Cassidy. An empirical and theoretical study of freeway wave bottlenecks, California PATH program, University of California, Berkeley, 70p, 2009.

P. Lertworawanich and L. Elefteriadou. A methodology for estimating capacity at ramp weaves based on gap acceptance and linear optimization. Transportation Research Part B: Methodological, 37(5):459-483, 2003.

F. Marczak and C. Buisson. Analytical derivation of capacity at diverging junctions. Transportation Research Board Annual Meeting, Paper 14-1625, 2014.

F. Marczak, W. Daamen, and C. Buisson. Merging behavior: Empirical comparison between two sites and new theory developments. Transportation Research Part C: Emerging Technologies, 36(0):530-546, 2013. 
F. Marczak, W. Daamen, and C. Buisson. Empirical analysis of lane changing behavior at a freeway weaving section. Transportation Research Board Annual Meeting, Paper 14-1097, 2014.

MOCoPo. Measuring and mOdelling Congestion and Pollution. http://www.mocopo.ifsttar.fr, 2011.

G.F. Newell. A simplified car-following theory: a lower order model. Transportation Research Part B: Methodological, 36(3):195-205, 2002.

M. Ng and S. Travis Waller. A dynamic route choice model considering uncertain capacities. Computer-Aided Civil and Infrastructure Engineering, 27(4):231-243, 2012.

D. Ngoduy. Derivation of continuum traffic model for weaving sections on freeways. Transportmetrica, 2(3): 199-222, 2006.

D. Ngoduy. Kernel smoothing method applicable to the dynamic calibration of traffic flow models. ComputerAided Civil and Infrastructure Engineering, 26(6):420-432, 2011.

H. Rakha and Y. Zhang. Analytical procedures for estimating capacity of freeway weaving, merge, and diverge sections. Journal of Transportation Engineering, 132(8):618-628, 2006.

P. Reina and S. Ahn. Prediction of merge ratio using lane flow distribution. Transportation Research Board Annual Meeting, Paper 14-5459, 2014.

R.P. Roess and J.M. Ulerio. Level of service analysis of freeway weaving segments. Transportation Research Record: Journal of the Transportation Research Board, 2130, pages 25-33, 2009a.

R.P. Roess and J.M. Ulerio. Capacity of freeway weaving segments. Transportation Research Record: Journal of the Transportation Research Board, 2130, pages 34-41, $2009 \mathrm{~b}$.

J. Rudjanakanoknad and C. Akaravorakulchai. Mechanism of a freeway weaving section as typical traffic bottleneck. Transportation Research Board Annual Meeting, Paper 11-0747, 2011.

M. Sarvi and M. Kuwahara. Microsimulation of freeway ramp merging processes under congested traffic conditions. IEEE Transactions on Intelligent Transportation Systems, 8(3):470-479, 2007.

M. Sarvi, O. Ejtemai, and A. Zavabeti. Modelling freeway weaving manoeuvre. In Australasian Transport Research Forum 2011 Proceedings, volume 34(0070), 16p, 2011.

A. Skabardonis. Simulation of freeway weaving areas. Transportation Research Record: Journal of the Transportation Research Board, 1802, pages 115-124, 2002. 
A. Skabardonis and A.M. Kim. Weaving analysis, evaluation and refinement, California PATH program, University of California, Berkeley, 70p, 2010.

D. Sun and L. Elefteriadou. Lane-changing behavior on urban streets: an "in-vehicle" field experiment based study. Computer-Aided Civil and Infrastructure Engineering, 27(7):525-542, 2012.

J.M. Torné, F. Soriguera, and N. Geroliminis. On the consistency of freeway macroscopic merging model. Transportation Research Board Annual Meeting, Paper 14-0324, 2014.

TRB. Highway Capacity Manual (HCM), Washington D.C.: National Research Council, 2000.

TRB. Highway Capacity Manual (HCM), Washington D.C.: National Research Council, 2010.

G. Zhang and Y. Wang. Optimizing coordinated ramp metering: a preemptive hierarchical control approach. Computer-Aided Civil and Infrastructure Engineering, 28(1):22-37, 2013. 